\title{
Fatty Liver Linked to Reduced Frequency of Ocular Complications in T2DM
}

\author{
Comlan Jules Gninkoun 1* ${ }^{*}$, Sylvie A. Ahn'2, K. Daniel Amoussou-Guenou ${ }^{3}$, \\ Evariste Bouenizabila4, Michel F. Rousseau ${ }^{2}$, Michel P. Hermans ${ }^{5}$
}

\begin{abstract}
${ }^{1}$ Service d'Endocrinologie \& Diabétologie, Faculté des Sciences de la Santé, Cotonou, Bénin
${ }^{2}$ Division of Cardiology, Cliniques Universitaires St-Luc and Pôle de Recherche Cardiovasculaire, Institut de Recherche Expérimentale et Clinique (IREC), Université catholique de Louvain, Brussels, Belgium

${ }^{3}$ Departmental Hospital and University Centre, Service de Médecine interne-Endocrinologie, CHUD/OP Porto-Novo, Université d'Abomey-Calavi, Abomey-Calavi, Bénin

${ }^{4}$ Service de Maladies Métaboliques et Endocriniennes, Centre Hospitalier et Universitaire de Brazzaville, Brazzaville, Congo ${ }^{5}$ Division of Endocrinology \& Nutrition, Cliniques universitaires St-Luc and Institut de Recherche Expérimentale et Clinique (IREC), Université catholique de Louvain, Brussels, Belgium

Email: *julesla67@yahoo.fr
\end{abstract}

How to cite this paper: Gninkoun, C.J., Ahn, S.A., Amoussou-Guenou, K.D., Bouenizabila, E., Rousseau, M.F. and Hermans, M.P. (2020) Fatty Liver Linked to Reduced Frequency of Ocular Complications in T2DM. Journal of Diabetes Mellitus, 10, 154-168.

https://doi.org/10.4236/jdm.2020.103013

Received: June 4, 2020

Accepted: August 9, 2020

Published: August 12, 2020

Copyright $\odot 2020$ by author(s) and Scientific Research Publishing Inc. This work is licensed under the Creative Commons Attribution International License (CC BY 4.0).

http://creativecommons.org/licenses/by/4.0/

\begin{abstract}
Purpose: Non-alcoholic fatty liver (NAFL) is comorbid to obesity, metabolic syndrome and type 2 diabetes mellitus (T2DM). It is unclear whether NAFLD constitutes a direct risk factor for macrovascular disease in T2DM. This study aimed at revisiting the cardiometabolic traits linked to NADL and micro-/ macrovascular complications in a biethnic Caucasian and African cohort. Methods: Cross-sectional analysis of 568 of T2DM patients (515 Caucasians; 53 Africans) in whom the presence of NAFL was identified by ultrasonography and the cardiometabolic phenotype was exhaustively characterized, including carbohydrate homeostasis, comprehensive lipidogram including Lp(a), cumulative exposure to hyperglycemia, and prevalent micro/macrovascular complications. Results: FL was present in $73 \%$ of Caucasians and $36 \%$ of Africans (p $<0.0001)$. FL+ were more obese, more atherogenic dyslipidemic (Caucasians) and had lower lipoprotein(a) (Africans). All-cause macroangiopathy, ischemic heart disease or stroke did not significantly differ between FL+ and FL- in both groups. A marked reduction in diabetic retinopathy (DR), ocular hypertonia and cataracts were found in FL+ of the two ethnicities. In FL+ Caucasians, relative risk of DR was $-38 \%$, cataracts $-35 \%$, and ocular hypertonia $-42 \%$. In FL+ Africans, risk of overall microangiopathy was $-66 \%$ and that of DR $-86 \%$. Conclusions: An inverse association was observed between NAFL and the presence of diabetic retinopathy, cataract and ocular hypertonia. The ophthalmoprotection conferred by liver steatosis was found in Caucasians and
\end{abstract}


Africans. Among the latter, hepatic steatosis was linked to lower lipoprotein(a) levels. There was no association between hepatic steatosis and prevalent macrovascular complications in neither of the two ethnic groups.

\section{Keywords}

Fatty Liver, Type 2 Diabetes, Microangiopathy, Retinopathy, Coronary Artery Disease, Lipoprotein(a)

\section{Introduction}

Non-alcoholic fatty liver (NAFL) is often comorbid to obesity, metabolic syndrome (MetS) and type 2 diabetes (T2DM). Regardless of glucose homeostasis, NAFL can develop into liver disease (NAFLD), i.e. non-alcoholic steatohepatitis (NASH), fibrosis, cirrhosis, liver failure and hepatocarcinoma. Epidemiologically, NAFL (D) is linked to unhealthy lifestyles and adverse cardiometabolic or cardiovascular (CV) phenotypes, and the presence of FL is predictive of incident $\mathrm{CV}$ diseases (CVD). While it is not clear whether NAFLD represents a direct risk factor in the development of macrovascular disease, suggested mechanisms include atherogenic dyslipidemia (AD), insulin resistance (IR), hepatokine and adipokine imbalance, systemic inflammation, hypercoagulation, hypofibrinolysis, and endothelial dysfunction [1] [2] [3] [4].

The link between NAFLD and the joint presence of organ-specific complications is poorly established in T2DM, particularly in terms of microvascular disease. This is compounded by the fact that, as for macrovascular disease, there are many confounding factors associated with diabetic microangiopathy and/or poor glycemic control, including as $\mathrm{AD}$ and other MetS components. Besides, considerable ethnic variations exist as regards NAFL prevalence or triglycerides (TG) and TG-rich lipoproteins (TRLs) metabolism, particularly among sub-Saharan Black africans [5]-[10].

We recently performed systematic non-invasive ultrasound research of NAFL for routine characterization of $\mathrm{CV}$ risk among outpatient diabetics, to revisit the cardiometabolic traits linked to NAFL and micro-/macrovascular complications. To detect a possible effect of ethno-geographic origin on these interactions, we analyzed a biethnic diabetic cohort composed of Caucasians and sub-Saharan Africans, the latter either $1^{\text {st }}$ generation migrants or expatriates living in Europe, for whom there is little or no relevant metabolic data on NAFL.

\section{Patients \& Methods}

This was a cross-sectional analysis of 578 medical records of T2DM outpatients (Caucasians $(\mathrm{n}=525)$ and sub-Saharan Africans $(\mathrm{n}=53)$ ) followed at an academic centre in Brussels. In all patients, a fatty liver screening was performed by ultrasound. A default diagnosis of simple uncomplicated liver steatosis (NAFL) 
was made in the presence of ultrasonographic hyper-reflectivity of the hepatic parenchyma (relative to the renal parenchyma), in the absence of dedicated etiologies of hepatic steatosis, such as excessive ethanol consumption and secondary causes of NAFL(D), the latter including inherited disorders of lipid metabolism; glycogen storage diseases; lipodystrophies; drugs (amiodarone; methotrexate; tamoxifen; nontopical corticosteroids; antiretrovirals); hepatitis $\mathrm{C}$ infection; celiac disease; Wilson's disease; surgical weight loss [11]. Patients with clinical, radiological or biopsy-proven evidence of liver cirrhosis were excluded, as were those treated with drugs that could affect insulin sensitivity (IS) or $\beta$-cell function, other than glucose-lowering drugs (e.g. corticosteroids, antiretrovirals, immune-modulators, anti-psychotics). Patients with chronic inflammatory or infectious diseases, ongoing infections and/or other acute conditions, cancer or major respiratory or cardiac failure were also excluded.

The following variables were recorded: age; gender; diabetes duration; education, as proxy for socio-economic status (dichotomized as lower vs. higher); smoking; ethanol intake; physical activity; weight; height; neck and waist circumference; body mass index (BMI); blood pressure (BP); fat mass, visceral fat and skeletal muscle mass (Omron BF 500, Omron Healthcare Europe B.V., Hoofddorp, The Netherlands). First-degree familial histories for T2DM and/or early-onset coronary heart disease (EOCHD) were also recorded. Hypertension was defined as systolic $\mathrm{BP} \geq 140$ and/or diastolic $\mathrm{BP} \geq 90 \mathrm{mmHg}$, and/or ongoing antihypertensive medication(s). Current therapies were recorded, including glucose-lowering drugs, BP-lowering therapies, and lipid-modifying drugs (LMDs: statins; fibrates and/or ezetimibe) and aspirin.

Methods used to define MetS, sleep apnoea, atherogenic dyslipidemia (AD), microangiopathies (diabetic retinopathy (DR); diabetic polyneuropathy (DPN)), and macrovascular complications (coronary artery disease (CAD); cerebrovascular disease [transient ischemic attack (TIA) and/or stroke]; peripheral artery disease (PAD)) were previously described [12] [13] [14] [15] [16]. Briefly, CAD was diagnosed from medical history (myocardial infarction, angioplasty, stenting, revascularization surgery and/or significant coronary stenosis confirmed by angiography), and review of all procedures, and/or screening (exercise testing; echocardiography; magnetic resonance imaging; or other subclinical disease imaging techniques). Cerebrovascular disease was defined as a history of stroke (any neurological deficit $\geq 1$ month, without distinction between ischemic, embolic and haemorrhagic events) and/or TIA. PAD was defined by a medical history of lower-limb(s) claudication and/or clinical or imaging evidence for ischemic diabetic foot, angioplasty, stenting, re-vascularization surgery and/or lower-limb artery stenosis at Doppler ultrasonography and/or angiography. The presence of a DR was established from retinal examination by an experienced ophthalmologist and/or fluorescein angiography. The presence of a DPN was diagnosed by clinical examination (knee and ankle reflexes; Semmes-Weinstein plantar and toe monofilament test) and confirmed by lower-limbs electromyography. The 
presence of cataracts and glaucoma was also noted. Microalbuminuria was considered for a urine albumin $(\mathrm{mg} / \mathrm{dL}) /$ creatinine $(\mathrm{g} / \mathrm{dL})$ ratio $>30 \mathrm{mg} / \mathrm{g}$. Selfreported prevalence/severity of masculine erectile dysfunction were identified using the 5-items International Index of Erectile Function (IIEF-5) questionnaire.

Fasting lipids and lipoproteins (total cholesterol (C), HDL-C, triglycerides (TG); LDL-C (calculated using Friedewald's formula), non-HDL-C, lipoprotein(a), apolipoproteins A-I and B100 were analysed by routine methods. HbA1c, fasting glucose, insulinemia, CRP, ferritin, sex hormone-binding globulin (SHBG), and liver enzymes aspartate aminotransferase, alanine aminotransferase, $\gamma$-glutamyl transferase were determined by routine methods. The aspartate aminotransferase-to-platelet ratio index (APRI) was also calculated, as was the hyperglycemia index (\%.year-1), the product of the difference between the annualized $\mathrm{HbA1c}$ of patients and the upper limit of normal for HbA1c (6\%) and diabetes duration.

$\beta$-cell function and insulin sensitivity (IS) were assessed with HOMA-2 modelling. Values of insulin secretion ([B]; normal 100\%) were plotted as a function of IS ([S]; normal 100\%), defining a hyperbolic product [BxS] (unit: \%2; normal: $100 \%$, corresponding to $104 \% 2$ ), representing residual $\beta$-cell function. Secular loss of hyperbolic product $([\mathrm{BxS}]$ loss rate; \%.year-1) was obtained by dividing (100\%-[BxS]) by patient's age [17] [18] [19] [20].

Each patient gave informed consent, and the study was performed in agreement with the principles of the Declaration of Helsinki and Good Clinical Practice. The study protocol was approved by the Biomedical Hospital-Faculty Ethics Committee of the Catholic University of Louvain (Brussels) B403-2017-16NOV-521. Results are presented as means ( \pm 1 standard deviation; SD) or proportions $(\%)$. The significance of differences between means was assessed using Student's t test, or Welch's test for data sets with significant differences in SDs, and using Fisher's Exact test for differences in proportions. Results were considered statistically significant or non-significant (NS) for $\mathrm{p}<0.05$ or $\mathrm{p} \geq 0.05$, respectively.

\section{Results}

\subsection{Characteristics of the Study Population}

Ten patients, all among Caucasians, with NASH diagnosed on the basis of hepatic biopsy and/or pathological fibroscan were excluded from further analysis. NAFL was present in $69 \%$ of the remaining patients $(n=393)$. NAFL prevalence was significantly different between Caucasians and Africans, with $73 \%$ frequency among Caucasians vs. $36 \%$ among Africans ( $\mathrm{p}<0.0001)$. For all patients $(\mathrm{n}=$ 568 ), average age was 70 (12) years; duration of diabetes was 19 (10) years; and gender distribution was predominantly male at 63\%. Lower and higher education levels were equally distributed, as was smoking history. Most patients were hypertensive and sedentary. Four-fifths received LMDs, mostly statins (Table 1). A majority of patients had sarcopenic obesity, enlarged waist, IR and a MetS. 
Table 1. Patients' characteristics.

\begin{tabular}{|c|c|c|c|c|c|c|c|c|}
\hline & & all patients & $\begin{array}{c}\text { FL[-] } \\
\text { Caucasian }\end{array}$ & $\begin{array}{c}\text { FL[+] } \\
\text { Caucasian }\end{array}$ & $P 1$ & $\begin{array}{c}\text { FL[-] } \\
\text { African }\end{array}$ & $\begin{array}{c}\text { FL[+] } \\
\text { African }\end{array}$ & $P 2$ \\
\hline$n$ & & 568 & 141 & 374 & $\sim$ & 34 & 19 & $\sim$ \\
\hline age & years & $70(12)$ & $73(13)$ & $70(11)$ & 0.0158 & $62(16)$ & $59(12)$ & $N S$ \\
\hline diabetes duration & years & $19(10)$ & $20(10)$ & $19(9)$ & $N S$ & $20(12)$ & $14(6)$ & 0.0190 \\
\hline family history T2DM & $\%$ & 48 & 47 & 48 & $N S$ & 45 & 47 & $N S$ \\
\hline family history EOCHD & $\%$ & 13 & 14 & 13 & $N S$ & 4 & 6 & $N S$ \\
\hline male - female & $\%$ & $63-37$ & $62-38$ & $64-36$ & $N S$ & $53-47$ & $53-47$ & $N S$ \\
\hline education* & $\%$ & $50-50$ & $49-51$ & $51-49$ & $N S$ & $52-48$ & $35-65$ & $N S$ \\
\hline smoking ${ }^{* *}$ & $\%$ & $50-50$ & $56-44$ & $44-56$ & 0.0176 & $81-19$ & $79-21$ & $N S$ \\
\hline ethanol & U.week ${ }^{-1}$ & $8(11)$ & $7(10)$ & $8(12)$ & $N S$ & $6(10)$ & $7(10)$ & $N S$ \\
\hline sedentarity & $\%$ & 62 & 53 & 68 & 0.0020 & 59 & 26 & $N S$ \\
\hline physical activity & $\min \cdot$ week $^{-1}$ & $58(111)$ & $74(125)$ & $47(99)$ & 0.0221 & $59(104)$ & $141(181)$ & $N S$ \\
\hline hypertension & $\%$ & 89 & 86 & 91 & $N S$ & 85 & 74 & $N S$ \\
\hline systolic blood pressure & $\mathrm{mm} \mathrm{Hg}$ & $138(19)$ & $140(20)$ & $138(19)$ & $N S$ & $135(15)$ & $132(16)$ & $N S$ \\
\hline anti-dyslipidemic drug(s) & $\%$ & 79 & 75 & 84 & 0.0206 & 53 & 44 & $N S$ \\
\hline statin - fibrate - ezetimibe & $\%$ & $70-21-13$ & $68-17-17$ & $74-26-11$ & $N S$ & $50-3-15$ & $44-0-6$ & $N S$ \\
\hline eGFR & $\mathrm{mL} \cdot \mathrm{min}^{-1} .1 .73 \mathrm{~m}^{2}$ & $74(28)$ & $70(26)$ & $74(27)$ & $N S$ & $85(37)$ & $98(25)$ & $N S$ \\
\hline
\end{tabular}

Results are expressed as means (1 SD) or proportions (\%) for all patients and according to the presence (FL[+]) or absence (FL[-]) of fatty liver at ultrasonography in patients of Caucasian or African ancestry. ${ }^{*}$ : lower vs. higher; ${ }^{* *}$ : never vs. former + current smoking; eGFR: estimated glomerular filtration rate; EOCHD: early-onset coronary heart disease; T2DM: type 2 diabetes mellitus; NS: not significant. P1 and P2 correspond to the statistical significance of the differences between the FL[-] and FL[+] subgroups among Caucasians and Africans, respectively.

One in six patients was diagnosed with sleep apnea. Glycaemic control was, on average, suboptimal with HbA1c 7.5 (1.4) \%. A majority of patients were treated with metformin and/or insulin (Table 2). Average values of hepatic enzymology were normal, as was mean APRI. Nearly half of patients had AD, the combination of lowHDL-C and high TG, and its prevalence was 52\% among Caucasians compared to $21 \%$ among Africans, ie a $60 \%$ reduction of $\mathrm{AD}$ frequency among the latter ( $\mathrm{p}<0.0001)$ (Table 3$)$. Regarding target organ damage, nearly half of patients had microvascular disease from all types, one-third had cataracts, and $13 \%$ had ocular hypertonia. Microalbuminuria or proteinuria was present in one-third of patients. More than half of men had erectile dysfunction. Macroangiopathy of all types was found in $37 \%$ of patients, including $27 \%$ CAD (Table 4).

\subsection{Caucasians with and without FL}

$\mathrm{FL}[+]$ were on average 3 years younger than FL[-] patients. They were more likely to be smokers and sedentary, and were more likely to be treated with LMDs. In patients of both groupes on fibrates, the drug was prescribed for atherogenic dyslipidemia, not as a secondary prevention of retinopathy. FL[-] and FL[+] did not differ in terms of family histories, gender distribution, education, alcohol consumption, prevalence of hypertension, and glomerular filtration (Table 1). 
Table 2. Cardiometabolic characteristics.

\begin{tabular}{|c|c|c|c|c|c|c|c|c|}
\hline & & all patients & $\begin{array}{c}\text { FL[-] } \\
\text { Caucasian }\end{array}$ & $\begin{array}{c}\text { FL }[+] \\
\text { Caucasian }\end{array}$ & $P 1$ & $\begin{array}{c}\text { FL }[-] \\
\text { African }\end{array}$ & $\begin{array}{c}\text { FL }[+] \\
\text { African }\end{array}$ & $P 2$ \\
\hline$n$ & & 568 & 141 & 374 & $\sim$ & 34 & 19 & $\sim$ \\
\hline body mass index & $\mathrm{kg} \cdot \mathrm{m}^{-2}$ & $29.1(5.7)$ & $26.1(5.1)$ & $30.4(5.6)$ & $<0.0001$ & $26.7(3.6)$ & $29.6(4.5)$ & 0.0220 \\
\hline skeletal muscle mass & $\%$ & $30.5(4.6)$ & $31.6(4.9)$ & $30.2(4.4)$ & 0.0033 & $30.3(5.4)$ & $29.5(5.7)$ & $N S$ \\
\hline fat mass & $\%$ & $32(9)$ & $29(9)$ & $33(9)$ & $<0.0001$ & $32(10)$ & $34(10)$ & $N S$ \\
\hline visceral fat & $0-30$ & $13(5)$ & $11(5)$ & $14(4)$ & $<0.0001$ & $11(3)$ & $11(4)$ & $N S$ \\
\hline waist circumference & $\mathrm{cm}$ & $104(14)$ & $97(14)$ & $107(13)$ & $<0.0001$ & $93(10)$ & $101(11)$ & 0.0130 \\
\hline waist.height $^{-1}$ & & $0.61(0.08)$ & $0.57(0.08)$ & $0.63(0.08)$ & $<0.0001$ & $0.55(0.06)$ & $0.59(0.05)$ & 0.0129 \\
\hline neck circumference & $\mathrm{cm}$ & $40(4)$ & $38(4)$ & $41(3)$ & $<0.0001$ & $38(3)$ & $39(3)$ & $N S$ \\
\hline sleep apnoea syndrome & $\%$ & 17 & 9 & 22 & 0.0002 & 3 & 6 & $N S$ \\
\hline metabolic syndrome & $\%$ & 86 & 70 & 94 & $<0.0001$ & 71 & 74 & $N S$ \\
\hline & score $0-5$ & $3.8(1.1)$ & $3.3(1.2)$ & $4.1(1.0)$ & $<0.0001$ & $3.0(1.0)$ & $3.4(1.3)$ & $N S$ \\
\hline insulin sensitivity & $\%$ & $51(37)$ & $74(48)$ & $41(27)$ & $<0.0001$ & $71(45)$ & $43(17)$ & 0.0022 \\
\hline fasting insulinaemia & $\mathrm{pmol} \cdot \mathrm{L}^{-1}$ & $123(84)$ & $87(66)$ & $139(89)$ & $<0.0001$ & $93(61)$ & $124(52)$ & NS \\
\hline hyperbolic product $[\mathrm{B} \times \mathrm{S}]$ & $\%$ & $27(18)$ & $32(20)$ & $24(15)$ & $<0.0001$ & $40(26)$ & $39(19)$ & $N S$ \\
\hline$[\mathrm{B} \times \mathrm{S}]$ loss rate & $\% \cdot$ year $^{-1}$ & $1.31(0.50)$ & $1.21(0.52)$ & $1.37(0.47)$ & 0.0016 & $1.19(0.52)$ & $1.33(0.61)$ & $N S$ \\
\hline $\mathrm{HbA}_{1 \mathrm{c}}$ & $\%$ & $7.5(1.4)$ & $7.5(1.4)$ & $7.6(1.4)$ & NS & $7.5(2.0)$ & $7.0(1.4)$ & $N S$ \\
\hline hyperglycemia index & $\% \cdot$ year $^{-1}$ & $29(36)$ & $28(42)$ & $31(33)$ & NS & $30(37)$ & $12(17)$ & 0.0194 \\
\hline metformin & $\%$ & 66 & 55 & 71 & 0.0008 & 61 & 79 & $N S$ \\
\hline BCS - IBT - SGLT2-i & $\%$ & $44-14-5$ & $39-14-1$ & $47-14-7$ & $N S-N S-0.0091$ & $42-12-0$ & $14-7-0$ & $N S$ \\
\hline \multirow[t]{2}{*}{ insulin } & $\%$ & 52 & 51 & 54 & $N S$ & 36 & 29 & $N S$ \\
\hline & IU $\cdot$ day $^{-1}$ & $68(57)$ & $49(29)$ & $78(64)$ & $<0.0001$ & $41(24)$ & $47(30)$ & $N S$ \\
\hline
\end{tabular}

Results are expressed as means (1 SD) or proportions (\%) for all patients and according to the presence (FL[+]) or absence (FL[-]) of fatty liver at ultrasonography in patients of Caucasian or African ancestry. BCS: beta-cell stimulant; HbA1c: glycated hemoglobin A1c; IBT: incretin-based therapies; SGLT2-i: sodium-glucose transporter type 2 inhibitors; NS: not significant. P1 and P2 correspond to the statistical significance of the differences between the FL[-] and $\mathrm{FL}[+]$ subgroups among Caucasians and Africans, respectively.

Table 3. Laboratory values.

\begin{tabular}{|c|c|c|c|c|c|c|c|c|}
\hline & & all patients & $\begin{array}{c}\text { FL[-] } \\
\text { Caucasian }\end{array}$ & $\begin{array}{c}\text { FL[+] } \\
\text { Caucasian }\end{array}$ & $P 1$ & $\begin{array}{c}\text { FL[-] } \\
\text { African }\end{array}$ & $\begin{array}{c}\text { FL[+] } \\
\text { African }\end{array}$ & $P 2$ \\
\hline$n$ & & 568 & 141 & 374 & $\sim$ & 34 & 19 & $\sim$ \\
\hline total cholesterol (C) & $\mathrm{mg} \cdot \mathrm{dL}^{-1}$ & $162(40)$ & $162(38)$ & $160(42)$ & $N S$ & $177(33)$ & $185(39)$ & $N S$ \\
\hline non-HDL-C & $\mathrm{mg} \cdot \mathrm{dL}^{-1}$ & $115(39)$ & $111(34)$ & $115(41)$ & $N S$ & $116(38)$ & $127(37)$ & $N S$ \\
\hline LDL-C & $\mathrm{mg} \cdot \mathrm{dL}^{-1}$ & $84(34)$ & $86(32)$ & $81(34)$ & $N S$ & $97(34)$ & $104(35)$ & $N S$ \\
\hline lipoprotein(a) & $\mathrm{nmol} \cdot \mathrm{L}^{-1}$ & $59(80)$ & $54(64)$ & $53(81)$ & $N S$ & $141(101)$ & $90(49)$ & 0.0170 \\
\hline $\operatorname{apoB}_{100}$ & $\mathrm{mg} \cdot \mathrm{dL}^{-1}$ & $89(25)$ & $86(22)$ & $89(27)$ & $N S$ & $90(22)$ & $101(23)$ & $N S$ \\
\hline LDL-C. $\operatorname{apoB}_{100}{ }^{-1}$ & & $0.94(0.26)$ & $1.00(0.27)$ & $0.90(0.25)$ & 0.0002 & $1.07(0.20)$ & $1.01(0.17)$ & $N S$ \\
\hline HDL-C & $\mathrm{mg} \cdot \mathrm{dL}^{-1}$ & $47(16)$ & $51(19)$ & $44(14)$ & $<0.0001$ & $60(18)$ & $58(21)$ & $N S$ \\
\hline
\end{tabular}


Continued

\begin{tabular}{|c|c|c|c|c|c|c|c|c|}
\hline apoA-I & $\mathrm{mg} \cdot \mathrm{dL}^{-1}$ & $144(29)$ & $150(32)$ & $141(27)$ & 0.0034 & $159(24)$ & $159(46)$ & $N S$ \\
\hline triglycerides & $\mathrm{mg} \cdot \mathrm{dL}^{-1}$ & $163(130)$ & $129(68)$ & $184(149)$ & $<0.0001$ & $91(44)$ & $116(52)$ & $N S$ \\
\hline atherogenic dyslipidemia & $\%$ & 49 & 35 & 59 & $<0.0001$ & 15 & 32 & $N S$ \\
\hline $\log (\mathrm{TG}) \cdot \mathrm{HDL}-\mathrm{C}^{-1}$ & & $0.054(0.053)$ & $0.048(0.028)$ & $0.059(0.062)$ & 0.0059 & $0.035(0.013)$ & $0.040(0.015)$ & $N S$ \\
\hline CRP & $\mathrm{mg} \cdot \mathrm{dL}^{-1}$ & $0.35(0.46)$ & $0.28(0.37)$ & $0.38(0.50)$ & 0.0140 & $0.29(0.30)$ & $0.39(0.54)$ & $N S$ \\
\hline ferritin & $\mu g \cdot L^{-1}$ & $157(163)$ & $130(171)$ & $168(163)$ & 0.0236 & $149(144)$ & $150(117)$ & $N S$ \\
\hline SHBG & $\mathrm{nmol} \cdot \mathrm{L}^{-1}$ & $41(30)$ & $47(30)$ & $36(25)$ & 0.0001 & $63(67)$ & $48(29)$ & $N S$ \\
\hline AST & $\mathrm{IU} \cdot \mathrm{l}^{-1}$ & $26(15)$ & $22(8)$ & $28(17)$ & $<0.0001$ & $23(12)$ & $28(19)$ & $N S$ \\
\hline ALT & $\mathrm{IU} \cdot \mathrm{l}^{-1}$ & $31(24)$ & $23(11)$ & $34(28)$ & $<0.0001$ & $24(16)$ & $31(22)$ & $N S$ \\
\hline GGT & $\mathrm{IU} \cdot \mathrm{I}^{-1}$ & $47(60)$ & $37(45)$ & $51(67)$ & 0.0067 & $37(26)$ & $55(59)$ & $N S$ \\
\hline APRI & IU. $1^{-1} \cdot 10^{9-1}$ & $0.23(0.20)$ & $0.19(0.12)$ & $0.25(0.22)$ & $<0.0001$ & $0.22(0.10)$ & $0.30(0.38)$ & $N S$ \\
\hline
\end{tabular}

Results are expressed as means (1 SD) or proportions (\%) for all patients and according to the presence (FL[+]) or absence (FL[-]) of fatty liver at ultrasonography in patients of Caucasian or African ancestry. ALT: alanine aminotransferase; apo: apolipoprotein; APRI: AST to platelet ratio index; AST: aspartate aminotransferase; CRP: C-reactive protein; GGT: gamma-glutamyl transpeptidase; HDL: high-density lipoprotein; LDL: low-density lipoprotein; TG: triglycerides; SHBG: sex hormone-binding globulin; NS: not significant. P1 and P2 correspond to the statistical significance of the differences between the $\mathrm{FL}[-]$ and $\mathrm{FL}[+]$ subgroups among Caucasians and Africans, respectively.

Table 4. Target organ damage.

\begin{tabular}{|c|c|c|c|c|c|c|c|c|}
\hline & & all patients & $\begin{array}{c}\text { FL[-] } \\
\text { Caucasian }\end{array}$ & $\begin{array}{c}\text { FL[+] } \\
\text { Caucasian }\end{array}$ & $P 1$ & $\begin{array}{c}\text { FL[-] } \\
\text { African }\end{array}$ & $\begin{array}{c}\text { FL[+] } \\
\text { African }\end{array}$ & $P 2$ \\
\hline$n$ & & 568 & 141 & 374 & $\sim$ & 34 & 19 & $\sim$ \\
\hline microangiopathy & $\%$ & 46 & 50 & 46 & $N S$ & 50 & 17 & 0.0185 \\
\hline retinopathy & $\%$ & 24 & 32 & 20 & 0.0048 & 42 & 6 & 0.0093 \\
\hline polyneuropathy & $\%$ & 26 & 29 & 25 & $N S$ & 28 & 17 & $N S$ \\
\hline cataract & $\%$ & 30 & 40 & 26 & 0.0024 & 47 & 22 & $N S$ \\
\hline glaucoma & $\%$ & 13 & 19 & 11 & 0.0403 & 13 & 0 & $N S$ \\
\hline albuminuria & mg.g creatinine ${ }^{-1}$ & $132(436)$ & $165(549)$ & $127(414)$ & $N S$ & $121(192)$ & $9(8)$ & 0.0018 \\
\hline (micro) albuminuria & $\%$ & 32 & 33 & 34 & $N S$ & 34 & 0 & 0.0023 \\
\hline erectile dysfunction & $\%$ & 55 & 59 & 55 & $N S$ & 44 & 33 & $N S$ \\
\hline IIEF5 score & $1-25$ & $13(8)$ & $11(8)$ & $13(8)$ & 0.0456 & $17(7)$ & $22(2)$ & 0.0100 \\
\hline macroangiopathy & $\%$ & 37 & 40 & 39 & $N S$ & 18 & 11 & $N S$ \\
\hline $\begin{array}{c}\text { coronary artery } \\
\text { disease }\end{array}$ & $\%$ & 27 & 30 & 28 & $N S$ & 9 & 6 & $N S$ \\
\hline $\begin{array}{l}\text { cerebrovascular } \\
\text { disease }\end{array}$ & $\%$ & 9 & 11 & 9 & $N S$ & 3 & 6 & $N S$ \\
\hline $\begin{array}{c}\text { peripheral artery } \\
\text { disease }\end{array}$ & $\%$ & 11 & 13 & 10 & $N S$ & 9 & 0 & $N S$ \\
\hline
\end{tabular}

Results are expressed as means (1 SD) or proportions (\%) for all patients and according to the presence (FL[+]) or absence (FL[-]) of fatty liver at ultrasonography in patients of Caucasian or African ancestry. IIEF5: 5-item version of the International Index of Erectile Function questionnaire; NS: not significant. P1 and P2 correspond to the statistical significance of the differences between the FL[-] and FL[+] subgroups among Caucasians and Africans, respectively. 
All the anthropophysical and metabolic parameters studied differed considerably and significantly between groups, $\mathrm{FL}[+]$ being larger, fatter, more centrally obese and less muscular, and more often suffering from MetS and sleep apnea than FL[-]. FL[+] were significantly more resistant to insulin (+80\%). Their fasting insulinemia was increased $(+60 \%)$, and their residual insulin secretion, estimated by the $[\mathrm{BxS}]$ product, was reduced by $25 \%$, with higher annual loss rate of the latter $(+13 \%)$. FL[+] were more often treated with metformin or SGLT2-i, and their daily insulin doses were higher (+59\%). The two groups did not differ in terms of HbAlc and in lifetime glucose exposure, estimated by the hyperglycaemia index (Table 2).

Total cholesterol, non-HDL-C, LDL-C, lipoprotein(a) and apoB100 did not differ between FL[-] and FL[+]. On the other hand, all lipid parameters related to $\mathrm{AD}$ were significantly different between $\mathrm{FL}[-]$ and $\mathrm{FL}[+]$, the latter also showing smaller and denser LDLs, as shown by a decreased LDL-C to apoB100 ratio. FL[+] also had increased CRP, ferritin, AST, ALT, GGT, and APRI levels, and decreased SHBG (Table 3).

In terms of vascular and avascular complications, $\mathrm{FL}[+]$ had markedly and significantly lower rates of DR (-38\%) and cataracts (-35\%), as well as ocular hypertonia $(-42 \%)$. There was no significant difference between FL[+] and FL[-] regarding albuminuria, whereas erectile dysfunction (ED) was slightly less prevalent, but not significantly so in FL[+], whose mean IIEF5 score was 2 units higher $(+18 \%)$. However, there was no difference in frequency of macrovascular complications between groups, regarding all-type macroangiopathy or site-specific damage to coronary, cerebrovascular or peripheral vessels (Table 4). This lack of difference in macrovascular complications based on FL persisted after adjusting the two groups for age and gender, with all-type macroangiopathy at $39 \%$ in FL[-] and $41 \%$ in FL[+] (not shown).

Since AD and FL are overlapping comorbidities, we analyzed whether their combined occurrence alters the prevalence of DR. Caucasians were distributed as follows: $42 \% \mathrm{FL}[+]$ \& $\mathrm{AD}[+] ; 30 \% \mathrm{FL}[+]$ \& $\mathrm{AD}[-] ; 18 \% \mathrm{FL}[-]$ \& $\mathrm{AD}[-]$; and $10 \% \mathrm{FL}[-] \& \mathrm{AD}[+]$, with $\mathrm{DR}$ prevalence of $21 \%$; $17 \%$; $27 \%$; and $42 \%$, respectively. All-type macroangiopathy was $43 \%$ in $\mathrm{FL}[+] \& \mathrm{AD}[+] ; 32 \%$ in $\mathrm{FL}[+] \&$ $\mathrm{AD}[-] ; 37 \%$ in $\mathrm{FL}[-] \& \mathrm{AD}[-]$; and $46 \%$ in $\mathrm{FL}[-] \& \mathrm{AD}[+]$. The presence of $\mathrm{AD}$ increased the relative risk of macroangiopathy among FL[+] by $34 \%$ (p 0.0308), whereas $\mathrm{AD}$ did not modulate macroangiopathy among $\mathrm{FL}[-]$ (data not shown).

\subsection{Africans with and without FL}

African were about 10 years younger than Caucasians and, as for the latter, FL[+] were younger than $\mathrm{FL}[-]$. On the other hand, diabetes duration was much shorter in $\mathrm{FL}[+]$, by an average of 6 years compared with FL[-]. All other variables (socio-demographics, BP, LMDs and eGFR) did not differ between FL[-] and FL[+] (Table 1). FL[+] had higher BMI, waist circumference and waist/height ratio. Their insulin sensitivity and hyperglycemia index were lower by $39 \%$ and $60 \%$, respectively (Table 2). AD prevalence was non-significantly higher (by 213\%) 
among FL[+]. None of the other variables concerning lipids and lipoproteins differed among Africans with or without FL, but for lipoprotein(a), the level of which was much lower among FL $[+]\left(-36 \mathrm{nmol} \cdot \mathrm{L}^{-1}\right.$; Table 3$)$.

As regards vascular and avascular complications, $\mathrm{FL}[+]$ had much lower rates of overall microangiopathy $(-66 \%)$ and DR $(-86 \%)$. There was no significant difference between groups regarding ocular hypertony and erectile dysfunction frequency, although as for Caucasians, FL[+] had better IIEF5 score (+29\%). FL[+] also showed much lower rates/level values of albuminuria. By contrast, there were no significant differences between $\mathrm{FL}[-]$ and $\mathrm{FL}[+]$ in overall macroangiopathy and damage to coronary, cerebrovascular or peripheral vessels (Table 4).

\section{Discussion}

This study conducted in a bi-ethnic population with T2DM revealed three highlights. First, there was no difference in prevalence of macrovascular disease between patients with and without FL in either ethnic group. Second, Lp(a) levels were markedly lower among steatotic Africans. Third, there was a marked reduction in prevalence of retinopathy, cataracts and ocular hypertonia in diabetics with steatosis, a lowering found in both ethnicities.

Our caracterization of steatotic Caucasians found a series of features which were expected based on published data, including physical inactivity, increased BMI, body fat, central adiposity, and sarcopenia [1] [2] [3] [4]. Caucasians with FL also had reduced insulin sensitivity, less residual insulin secretion, and more B-cell function loss. On the other hand, the prevalence of NAFL in sub-Saharan Africans is barely documented, even less so in diabetics. While data exist from across the Atlantic on African-Americans or Afro-Caribbean people, these minorities do have a certain degree of European, Indian or Latino crossbreeding that can modulate their metabolic features [5]-[10]. The present study, albeit numericaly modest, is the first to document in depth the steatotic phenotype of $1^{\text {st }}$ generation African migrants with diabetes.

An increased frequency of macroangiopathies was intuitively expected in steatotic patients, due to many overlaying $\mathrm{CV}$ risk factors, such as sedentary, smoking, raised $\mathrm{BMI}$, waist circumference, central adiposity, $\mathrm{AD}$, and subclinical inflammation. It is questionable whether these risk factors are as harmful in case of FL as they are in its absence, or whether the presence of simple steatosis mitigates, through unknown mechanisms, the deleterious exposure to these risk factors. It is not excluded that the longitudinal association observed between NAFL and incident macrovascular disease is more a result of overlapping epidemiological traits than a direct pathological link.

Our data clearly show a marked reduction in prevalence of retinopathy associated with the presence of FL never before described for Caucasians and Africans. This under-prevalence was occasionaly noted in Asians, whereas an overprevalence of retinopathy was reported by Targher et al. in Italians [21] [22] [23] [24]. Our data are the first to document an FL-related ophthalmoprotection in 
Caucasians and Africans, prevailing beyond the retinal vessels since ocular hypertonia and cataracts were also reduced. These two avascular eye disorders are not de facto caused by hyperglycemia, and such multiple organ protection ought to be studied prospectively in different ethnic groups [25]. While lifetime glycemic exposure could be evoked to account for the lower prevalence of retinopathy among steatotic Africans, whose hyperglycemia index was much lower due to better glycemic control and a shorter diabetes duration, this was not the case among Caucasians, in whom the $38 \%$ reduction in retinopathy related to FL cannot be ascribed to lesser hyperglycemia.

FL-related differences between ethnic groups were most evident at the lipid level, with a strong association between FL and AD among Caucasians, in whom AD prevalence and lipid indices point to more TRLs, small-dense LDLs, and reduced HDLs. These was not the case among steatotic Africans. Although they had more AD, FL+ Africans had still a lower AD prevalence than Caucasians without FL, confirming the marked ethnic dimorphism of TG handling among sub-Saharan Africans [6].

$\mathrm{AD}$ is epidemiologically associated with the presence of FL and micro-angiopathy, including DR, and causally associated with macroangiopathy [26] [27]. It would therefore be expected that the combined presence of $\mathrm{AD}$ and $\mathrm{FL}$ further increases vascular risk. This was indeed the case for all-cause macro-angiopathy. However, the opposite was observed for microangiopathy, with the lowest prevalence of $\mathrm{DR}$ observed in $\mathrm{FL}[+] \& \mathrm{AD}[+]$ patients, whereas the highest $\mathrm{DR}$ rate was observed in FL[-] \& $\mathrm{AD}[+]$, twice as high as that of FL[+] \& $\mathrm{AD}[+]$ patients ( $\mathrm{p}$ 0.0033; not shown). This shows that $\mathrm{AD}$ has a permissive effect on the development of retinal microangiopathy only in the absence of FL. By contrast, FL was associated with a significantly reduced prevalence of DR, regardless of the presence of AD. Due to the low prevalence of both FL and AD in sub-Saharan patients and limited number of patients, it was not feasible to analyze their combined effects in this study.

The presence of FL did not influence $\mathrm{Lp}(\mathrm{a})$ levels in Caucasians. While it was expected that $\mathrm{Lp}(\mathrm{a})$ would be higher among Africans, a known racial difference, our data show a new and previously unreported feature of this lipoprotein, namely an ethnic dimorphism as a function of FL. Thus, the latter was associated with lower Lp(a) among Blacks but not among Caucasians. One could suggest a possible link between lower $\mathrm{Lp}(\mathrm{a})$ level observed in steatotic Africans and reduced frequency of all-type microangiopathy and retinopathy. However, this was not observed among steatotic Caucasians, in whom we previously reported that lower $\mathrm{Lp}(\mathrm{a})$ was associated with increased prevalence of microvascular damage [14].

To explain the paradox of ophthalmoprotection related to FL, various hypotheses can be put forward. The least plausible is that having retinopathy would prevent the development of hepatic steatosis. There is nothing in the accepted pathophysiology of DR and FL to substantiate such assumption [28] [29]. Inversely, certain mechanisms promoting hepatic steatosis may be retinoprotective. 
The retina is an extension of the brain with a distinct metabolism of cholesterol and lipoproteins. Liver X receptor (LXR) signalling pathways could be involved in such a candidate process. In diabetes, decreased LXR and sirtuin-1 (an LXR agonist) could promote retinopathy while protecting against the development of liver steatosis. Conversely, endogenous or pharmacological activation of LXRs could exert a retinoprotective effect while promoting hepatic steatogenesis [30] [31] [32] [33].

This study has the limitations inherent to its cross-sectional design and small sample size, especially of Africans. This did not allow statistical thresholds to be reached for some differences that were otherwise evident among steatotic Africans. Given the small number of Africans, our results should be interpreted in an exploratory way, pending external confirmation. Patients were dichotomized based on a single ultrasound assessment of steatosis, and we could not distinguish, in the absence of liver biopsy or non-invasive fibrosis tests, patients with simple steatosis from those with frank NAFLD. We excluded cirrhotic patients and those with biopsy-proven NASH and/or overtly pathological fibroscan, but we cannot rule out that some patients had steatohepatitis, liver fibrosis, or incipient cirrhosis. However, APRI values show that the vast majority of patients had basic uncomplicated NAFL. One strength of the study is that it used an index of cumulative glucose exposure to better understand the relative microangiopathic risk of the two ethnicities, whose carbohydrate and lipid homeostases determinants are known as distinct.

\section{Conclusion}

In conclusion, our bi-ethnic analysis of T2DM patients did not show a connection between hepatic steatosis and macrovascular complications. Hepatic steatosis was linked to lower lipoprotein(a) levels only among Africans. In a serendipitous manner, an inverse and unexpected association was observed between diabetic retinopathy and two avascular eye complications, on the one hand, and the presence of hepatic steatosis, on the other hand. The ophthalmoprotection conferred by liver steatosis was found in Caucasians and Africans.

\section{Authors' Contributions}

All authors contributed equally to the manuscript; all authors read and approved the final version of the manuscript.

\section{Conflicts of Interest}

The authors declare no conflicts of interest regarding the publication of this paper.

\section{Informed Consent}

Informed consent was obtained from all individual participants included in the study. 
The study protocol was approved by the Biomedical Hospital-Faculty Ethics Committee of the Catholic University of Louvain (Brussels) B403-2017-16NOV-521.

\section{Availability of Data \& Material}

Data are available at the Division of Endocrinology \& Nutrition, Cliniques universitaires St-Luc and the Institut de Recherche Expérimentale et Clinique (IREC), Université catholique de Louvain, Brussels (Belgium) (person of contact: Prof. MP Hermans).

\section{References}

[1] Diehl, A.M. and Day, C. (2017) Cause, Pathogenesis, and Treatment of Nonalcoholic Steatohepatitis. The New England Journal of Medicine, 377, 2063-2072. https://doi.org/10.1056/NEJMra1503519

[2] Stefan, N., Häring, H.U. and Cusi, K. (2019) Non-Alcoholic Fatty Liver Disease: Causes, Diagnosis, Cardiometabolic Consequences, and Treatment Strategies. The Lancet Diabetes \& Endocrinology, 7, 313-324. https://doi.org/10.1016/S2213-8587(18)30154-2

[3] Targher, G., Day, C.P. and Bonora, E. (2010) Risk of Cardiovascular Disease in Patients with Nonalcoholic Fatty Liver Disease. The New England Journal of Medicine, 363, 1341-1350. https://doi.org/10.1056/NEJMra0912063

[4] Hagström, H., Nasr, P., Ekstedt, M., Hammar, U., Stål, P., Askling, J., Hultcrantz, R. and Kechagias, S. (2019) Cardiovascular Risk Factors in Non-Alcoholic Fatty Liver Disease. Liver International, 39, 197-204. https://doi.org/10.1111/liv.13973

[5] Onyekwere, C.A., Ogbega, A.O. and Balogun, B.O. (2011) Non-Alcoholic Fatty Liver Disease and the Metabolic Syndrome in an Urban Hospital Serving an Africa Community. Annals of Hepatology, 10, 119-124. https://doi.org/10.1016/S1665-2681(19)31559-5

[6] Yu, S.S., Castillo, D.C., Courville, A.B. and Sumner, A.E. (2012) The Triglyceride Paradox in People of African Descent. Metabolic Syndrome and Related Disorders, 10, 77-82. https://doi.org/10.1089/met.2011.0108

[7] Olusanya, T.O., Lesi, O.A., Adeyomoye, A.A. and Fasanmade, O.A. (2016) Non Alcoholic Fatty Liver Disease in a Nigerian Population with Type II Diabetes Mellitus. The Pan African Medical Journal, 24, 20. https://doi.org/10.11604/pamj.2016.24.20.8181

[8] Sherif, Z.A., Saeed, A., Ghavimi, S., Nouraie, S.M., Laiyemo, A.O., Brim, H. and Ashktorab, H. (2016) Global Epidemiology of Nonalcoholic Fatty Liver Disease and Perspectives on US Minority Populations. Digestive Diseases and Sciences, 61, 1214-1225. https://doi.org/10.1007/s10620-016-4143-0

[9] Bril, F., Portillo-Sanchez, P., Liu, I.C., Kalavalapalli, S., Dayton, K. and Cusi, K. (2018) Clinical and Histologic Characterization of Nonalcoholic Steatohepatitis in African American Patients. Diabetes Care, 41, 187-192. https://doi.org/10.2337/dc17-1349

[10] Naran, N.H., Haagensen, M. and Crowther, N.J. (2018) Steatosis in South African Women: How Much and Why? PLoS ONE, 13, e0191388. https://doi.org/10.1371/journal.pone.0191388

[11] Kneeman, J.M., Misdraji, J. and Corey, K.E. (2012) Secondary Causes of Nonalcoholic Fatty Liver Disease. Therapeutic Advances in Gastroenterology, 5, 199-207. https://doi.org/10.1177/1756283X11430859 
[12] Alberti, K.G., Eckel, R.H., Grundy, S.M., Zimmet, P.Z., Cleeman, J.I., Donato, K.A., Fruchart, J.C., James, W.P., Loria, C.M. and Smith Jr., S.C. (2009) Harmonizing the Metabolic Syndrome: A Joint Interim Statement of the International Diabetes Federation Task Force on Epidemiology and Prevention; National Heart, Lung, and Blood Institute; American Heart Association; World Heart Federation; International Atherosclerosis Society; and International Association for the Study of Obesity. Circulation, 120, 1640-1645. https://doi.org/10.1161/CIRCULATIONAHA.109.192644

[13] Hermans, M.P., Ahn, S.A., Amoussou-Guenou, K.D. and Rousseau, M.F. (2010) Impact of Metabolic Syndrome on Microvascular Complications in Type 2 Diabetes. Diabetology \& Metabolic Syndrome, 4, 150-154. https://doi.org/10.1016/j.dsx.2010.05.021

[14] Hermans, M.P., Ahn, S.A. and Rousseau, M.F. (2017) The Mixed Benefit of Low Lipoprotein(a) in Type 2 Diabetes. Lipids in Health and Disease, 16, 171.

https://doi.org/10.1186/s12944-017-0564-9

[15] Hermans, M.P., Amoussou-Guenou, K.D., Bouenizabila, E., Sadikot, S.S., Ahn, S.A. and Rousseau, M.F. (2017) Size, Density and Cholesterol Load of HDL Predict Microangiopathy, Coronary Artery Disease and $\beta$-Cell Function in Men with T2DM. Diabetology \& Metabolic Syndrome, 11, 125-131. https://doi.org/10.1016/j.dsx.2016.08.029

[16] Hermans, M.P., Mahadeb, Y.P., Katchunga, P., Cikomola Cirhuza, J., Ahn, S.A. and Rousseau, M.F. (2014) Novel Sexual Dimorphisms of Sleep Apnea Syndrome in Diabetes. Diabetology \& Metabolic Syndrome, 8, 36-44. https://doi.org/10.1016/j.dsx.2013.08.002

[17] Hermans, M.P., Levy, J., Morris, R.J. and Turner, R.C. (1999) Comparison of Insulin Sensitivity Tests across a Range of Glucose Tolerance from Normal to Diabetes. Diabetologia, 42, 678-687. https://doi.org/10.1007/s001250051215

[18] Hermans, M.P., Levy, J., Morris, R.J. and Turner, R.C. (1999) Comparison of Tests of Beta-Cell Function across a Range of Glucose Tolerance from Normal to Diabetes. Diabetes, 48, 1779-1786. https://doi.org/10.2337/diabetes.48.9.1779

[19] Munoko, Th. and Hermans, M.P. (2008) Phenotypic Characterization of First Generation Maghrebian Migrants with Type 2 Diabetes: A Gender-Based Comparison with a Reference North-Caucasian Belgian Cohort. Diabetology \& Metabolic Syndrome, 2, 115-124. https://doi.org/10.1016/j.dsx.2008.02.004

[20] Dehout, F., Haumont, S., Gaham, N., Amoussou-Guenou, K.D. and Hermans, M.P. (2008) Metabolic Syndrome in Bantu Subjects with Type 2 Diabetes from SubSaharan Extraction: Prevalence, Gender Differences and HOMA-Hyperbolic Product. Diabetology \& Metabolic Syndrome, 2, 5-11. https://doi.org/10.1016/j.dsx.2007.11.008

[21] Targher, G., Bertolini, L., Rodella, S., Zoppini, G., Lippi, G., Day, C. and Muggeo, M. (2008) Non-Alcoholic Fatty Liver Disease Is Independently Associated with an Increased Prevalence of Chronic Kidney Disease and Proliferative/Laser-Treated Retinopathy in Type 2 Diabetic Patients. Diabetologia, 51, 444-450. https://doi.org/10.1007/s00125-007-0897-4

[22] Lv, W.S., Sun, R.X., Gao, Y.Y., Wen, J.P., Pan, R.F., Li, L., Wang, J., Xian, Y.X., Cao, C.X. and Zheng, M. (2013) Nonalcoholic Fatty Liver Disease and Microvascular Complications in Type 2 Diabetes. World Journal of Gastroenterology, 19, 3134-3142. https://doi.org/10.3748/wjg.v19.i20.3134

[23] Kim, B.Y., Jung, C.H., Mok, J.O., Kang, S.K. and Kim, C.H. (2014) Prevalences of Diabetic Retinopathy and Nephropathy Are Lower in Korean Type 2 Diabetic Pa- 
tients with Non-Alcoholic Fatty Liver Disease. Journal of Diabetes Investigation, 5, 170-175. https://doi.org/10.1111/jdi.12139

[24] Yan, L.H., Mu, B., Guan, Y., Liu, X., Zhao, N., Pan, D. and Wang, S.Z. (2016) Assessment of the Relationship between Non-Alcoholic Fatty Liver Disease and Diabetic Complications. Journal of Diabetes Investigation, 7, 889-894.

https://doi.org/10.1111/jdi.12518

[25] Hermans, M.P., Ahn, S.A. and Rousseau, M.F. (2011) Statin Therapy and Cataract in Type 2 Diabetes. Diabetes \& Metabolism, 37, 139-143. https://doi.org/10.1016/j.diabet.2010.09.005

[26] Hermans, M.P., Ahn, S.A. and Rousseau, M.F. (2012) The Atherogenic Dyslipidemia Ratio [Log(TG)/HDL-C] Is Associated with Residual Vascular Risk, B-Cell Function Loss and Microangiopathy in Type 2 Diabetes Females. Lipids in Health and Disease, 11, 132. https://doi.org/10.1186/1476-511X-11-132

[27] Sacks, F.M., Hermans, M.P., Fioretto, P., Valensi, P., Davis, T., Horton, E., Wanner, C., Al-Rubeaan, K., Aronson, R., Barzon, I., Bishop, L., Bonora, E., Bunnag, P., Chuang, L.M., Deerochanawong, C., Goldenberg, R., Harshfield, B., Hernández, C., Herzlinger-Botein, S., Itoh, H., Jia, W., Jiang, Y.D., Kadowaki, T., Laranjo, N., Leiter, L., Miwa, T., Odawara, M., Ohashi, K., Ohno, A., Pan, C., Pan, J., Pedro-Botet, J., Reiner, Z., Rotella, C.M., Simo, R., Tanaka, M., Tedeschi-Reiner, E., Twum-Barima, D., Zoppini, G. and Carey, V.J. (2014) Association between Plasma Triglycerides and High-Density Lipoprotein Cholesterol and Microvascular Kidney Disease and Retinopathy in Type 2 Diabetes Mellitus: A Global Case-Control Study in 13 Countries. Circulation, 129, 999-1008. https://doi.org/10.1161/CIRCULATIONAHA.113.002529

[28] Hammer, S.S., Beli, E., Kady, N., Wang, Q., Wood, K., Lydic, T.A., Malek, G., Saban, D.R., Wang, X.X., Hazra, S., Levi, M., Busik, J.V. and Grant, M.B. (2017) The Mechanism of Diabetic Retinopathy Pathogenesis Unifying Key Lipid Regulators, Sirtuin 1 and Liver X Receptor. EBioMedicine, 22, 181-190. https://doi.org/10.1016/j.ebiom.2017.07.008

[29] Hammer, S.S. and Busik, J.V. (2017) The Role of Dyslipidemia in Diabetic Retinopathy. Vision Research, 139, 228-236. https://doi.org/10.1016/j.visres.2017.04.010

[30] Zheng, W., Reem, R.E., Omarova, S., Huang, S., DiPatre, P.L., Charvet, C.D., Curcio, C.A. and Pikuleva, I.A. (2012) Spatial Distribution of the Pathways of Cholesterol Homeostasis in Human Retina. PLoS ONE, 7, e37926.

https://doi.org/10.1371/journal.pone.0037926

[31] Ducheix, S., Montagner, A., Theodorou, V., Ferrier, L. and Guillou, H. (2013) The Liver X Receptor: A Master Regulator of the Gut-Liver Axis and a Target for Non Alcoholic Fatty Liver Disease. Biochemical Pharmacology, 86, 96-105. https://doi.org/10.1016/j.bcp.2013.03.016

[32] Xu, P., Zhai, Y. and Wang, J. (2018) The Role of PPAR and Its Cross-Talk with CAR and LXR in Obesity and Atherosclerosis. International Journal of Molecular Sciences, 19, e1260. https://doi.org/10.3390/ijms19041260

[33] Cave, M.C., Clair, H.B., Hardesty, J.E., Falkner, K.C., Feng, W., Clark, B.J., Sidey, J., Shi, H., Aqel, B.A., McClain, C.J. and Prough, R.A. (2016) Nuclear Receptors and Nonalcoholic Fatty Liver Disease. Biochimica et Biophysica Acta, 1859, 1083-1099. https://doi.org/10.1016/j.bbagrm.2016.03.002 


\section{Abbreviations}

AD: atherogenic dyslipidemia

Apo: apolipoprotein

APRI: aspartate aminotransferase-to-platelet ratio index

$[\mathrm{B} \times \mathrm{S}]$ : hyperbolic product between $\beta$-cell function and IS

BMI: body mass index

BP: blood pressure

CHD: coronary heart disease

CV: cardiovascular

CVD: cardiovascular disease

CRP: C-reactive proteion

DM: diabetes mellitus

eGFR: estimated glomerular filtration rate

EOCHD: early-onset CHD

FL: fatty liver

HbA1c: glycated haemoglobin

HDL: high-density lipoprotein

HDL-C: high-density lipoprotein cholesterol

HOMA: homeostasis model assessment

IBT: incretin-based therapies

IIEF5: 5-items International Index of Erectile Function

IS: insulin sensitivity

LDL: low-density lipoprotein

LDL-C: low-density lipoprotein cholesterol

LMD: lipid-modifying drug

MetS: metabolic syndrome

NAFL: non-alcoholic fatty liver

NAFLD: non-alcoholic fatty liver disease

non-HDL-C: non-high-density lipoprotein cholesterol

NS: non-significant

SD: standard deviation

T2DM: type 2 diabetes mellitus

TG: triglycerides (triacylglycerols)

SHBG: sex hormone-binding globulin 